\title{
Cognitive, Emotional, and Sociocultural Processes in Consumption
}

\author{
Chunyan Xie \\ Stord/Haugesund University College \\ Richard P. Bagozzi \\ University of Michigan
}

Jens Østli

Nofima Marked

\begin{abstract}
This article examines deliberative, emotional, and sociocultural processes in consumption. The authors draw upon basic processes from two leading theories in social psychology, the theory of planned behavior (TPB) and the model of goal-directed behavior (MGB), to develop a comprehensive approach to decision making more appropriate for many consumption decisions, and revise the representation and modeling of key variables to better reflect how social psychological processes relate to consumer behavior. A survey was conducted among real adult consumers of bacalhau in Portugal. Because it is most common for women to prepare bacalhau meals in Portugal, 153 female participants were recruited for this survey. The results show that the TPB, and especially the MGB, are found to explain food consumption decisions well but only after the approaches are modified in form and content to accommodate the complex emotional and social aspects of the consumption context. The results also show that the effects of key determinants of desire in the MGB are contingent on the traits of food involvement and cultural orientation (i.e., degree of vertical individualism). The approach taken herein overcomes limitations of existing theories by synthesizing relevant processes across two leading theories and by introducing new variables and processes, thereby showing that the organization of these processes and their contingency on cultural variables regulate consumption. (C) 2012 Wiley Periodicals, Inc.
\end{abstract}

Much of everyday consumption is mundane or easy to execute and entails decision making by an individual person. The product or service under consideration in such cases is typically functional or utilitarian or if not involves the assessment of such criteria as choice of options or attributes, when or where to make a purchase, quantity, how much to spend, etc. Alternatively, some everyday consumption is largely habitual, having been repeated often and even achieving the status of a ritual. These types of consumption have been studied fruitfully by applying the attitude model or other parsimonious frameworks (e.g., Armitage \& Conner, 2001; Dennison \& Sheppard, 1995; Verbeke \& Vackier, 2004).

Some everyday consumption decisions are more complex and involving and entail purposive consideration of a sequence of actions requiring anticipation of personal and sociocultural consequences, plus planning of multiple actions and contingencies. The current study is a case in point and examines complex consumption decisions of a specific dinner built around bacalhau, which is a culturally important fish dish in the country of study, Portugal. Bacalhau is a Portuguese word for a particular preparation of codfish and is synonymous with salted and dried cod nowadays. Bacalhau holds a special status in Portugal, both as a cultural "identity-tag" and very much appreciated foodstuff for special meals on weekdays and festivities (Østli, Heide, Carlehög, \& Eilertsen, 2006). Bacalhau consumption exhibits strong social, emotional, and cultural elements and contrasts with many other consumer choice situations where decisions are more individualistic, cognitive, and functional. Portuguese culture shares much with other Mediterranean cultures where warm interpersonal relationships and a family-centered life are nearly universal. It is the tradition in many Portuguese families for bacalhau consumption to serve as a centerpiece of family celebrations and social solidarity. Bacalhau dishes are so dominant in the Portuguese kitchen 
that cooking skills for bacalhau dishes are part of common folklore as a standard for matrimonial qualifications and family harmony.

To study and explain consumption of this sort, more subtle and refined approaches are required than found in standard attitude models (e.g., Astrøsm \& Rise, 2001; Bagozzi \& Natarajaan, 2000; Leone, Perugini, \& Ercolani, 2004; Sheeran \& Orbell, 1999; Zint, 2002). The present research is such an attempt. The purpose of this study is to adapt, extend, and deepen extant models and theories to explain consumption behavior, which involves rational, emotional, social, and cultural forces. A new integrative model of consumer behavior that lends itself to testing quantitative hypotheses in surveys is also introduced. This model goes beyond the modeling of rational process only, to include emotional, social, and cultural processes, as well as the effects of contingent individual differences.

The theory of planned behavior (TPB; Ajzen, 1991) is the starting point of this study. For the past three decades, the most frequently applied approach in survey research in consumer behavior has been the Theory of Reasoned Action (TRA) and its extension, the TPB. The TPB (e.g., Ajzen, 1991) has been applied successfully in hundreds of studies in the managerial, social, and health sciences (e.g., Armitage \& Conner, 2001). However, the TRA and TPB can be criticized for failing to incorporate emotional, social, and cultural processes. If one wishes to study and forecast consumer behaviors that entail rational as well as nonrational processes, especially in the context of family consumption, the TRA and TPB must be modified and expanded to incorporate affective and social processes.

As a consequence, a recent extension of the TPB, the model of goal-directed behavior (MGB), is drawn upon in the current study. The MGB was pioneered in Great Britain and which explicitly introduces emotional processes in a goal-directed framework (Perrugini \& Bagozzi, 2001; Perrugini \& Conner, 2000). The MGB broadens and deepens the TPB by including positive and negative anticipated emotions to account for affective forces in decision making. The MGB also posits that desire, another affective construct with special motivational and conative properties, mediates the effects of attitudinal antecedents and emotions on intentions. However, it should be noted that the MGB was developed in psychology and has not yet been used much in marketing (cf., Bagozzi \& Dholakia, 2006). Also the MGB has not been applied yet in family decision making settings. Moreover, the MGB still needs to be adapted to this consumer behavior context to reach its full potential, for it does not consider social processes, culture or contingencies. One of the tasks of this study is to perform such an adaptation. The MGB has little to say about social processes, yet there is reason to believe that some consumer decisions, such as bacalhau consumption are infused with special social meaning. Therefore, a new variable (i.e., social identity) is introduced into the MGB to address small-group based social processes in ba- calhau consumption (see rational below under section, Social Identity). Social identity has not been considered much in attitudinal research to date (cf., Bagozzi \& Dholakia, 2006; Povey, Conner, Sparks, James, \& Shepherd, 2000).

Different theories are tested and compared to explain decision making in this specific family consumption context. Moderating effects of two additional variables (i.e., cultural orientations and degree of involvement with food choice and preparation in decision making) are also examined. It should be pointed out that the TPB and the MGB are linear models and do not test contingencies. One of the goals of this study is to develop a theory that accounts for contingency and explains behavior that the TPB and MGB cannot explain in their current forms.

To sum up, the aim of this study is to investigate a type of complex consumption in a specific marketing context, bacalhau consumption, where social, emotional, and cultural forces are especially salient. Existing theories (i.e., the TPB and the MGB) are extended and deepened to take into account emotional and social processes in consumption, and then, further steps are taken to test possible influences of cultural orientation on decision making. Although the focus is on bacalhau consumption in order to provide a specific enough empirical tests for the new theory developed here, it is reasonable to believe that the general theory should fit such other social, emotionally, and culturally charged situations as family car purchases and holiday planning. Further, the approach would seem applicable to such group consumption decisions as choice of restaurant or recreational venues, among other contexts.

\section{THEORETICAL BACKGROUND}

As background, key aspects of the two baseline theoretical models applied in this study, the TPB and MGB, are first developed. This is done to provide a foundation for the integrative theory proposed herein.

\section{The TPB and the Limited Sense It Incorporates Social Criteria}

The TPB is a parsimonious and robust model and posits that attitudes toward an act (which are evaluative judgments made by individuals), felt subjective normative pressure to act, and perceived behavior control over an act determine one's intentions to act, which, in turn, influence behavior, together with actual control over one's behavior. Under the TPB, subjective norms (SN) refer to the perceived social pressure to perform or not to perform the behavior; the implied compliance processes are based on one's need for approval. It should be stressed that felt normative pressure captures only one type of social process: namely, one form of interpersonal processes. It does not incorporate what Kelman (1974) terms social identification processes and 
internalization processes, which can be important when people are members of a group (more on this below).

It might be argued further that the TPB's parsimony is also its weakness. It lacks the capacity to account for either emotional elements or social processes other than compliance, which as argued below are central in many sociocultural consumption situations. This was a major impetus for our inclusion of the MGB in the current study.

\section{The MGB and Anticipated Emotions, Desire, and Past Behavior}

Under the MGB, decisions are driven directly by the desire to act which functions as the central motivational process transforming reasons for acting (rational, emotional, and social) into actual decisions to act. The antecedents of desires include anticipated positive and negative emotions that one feels would transpire if one were to act, past behavior ( $\mathrm{PB}$ ), and the predictors under the TPB (i.e., attitudes, SN, and perceived behavioral control $[\mathrm{PBC}])$.

Anticipated Emotions. Anticipated positive and negative emotions have been found to be important determinants of decisions in a number of studies (e.g., Bagozzi \& Dholakia, 2006; Bagozzi, Baumgartner, \& Pieters, 1998; Perrugini \& Bagozzi, 2001; Perrugini \& Conner, 2000). The expression, experience, and sharing of emotions are important everyday occurrences for people of many cultures. Bagozzi, Baumgartner, and Pieters (1998) proposed a comprehensive approach to the role of emotions in decision making for goal-directed behaviors. They argued that people take into account the emotional consequences of both achieving and not achieving a goal when they consider whether nor not to act in goal-directed situations. The processes are believed to be predicated upon a type of thought process analogous to counterfactual thinking, but more accurately termed "prefactual appraisals" herein, whereby a decision maker imagines the affective consequences of goal attainment and goal failure before forming an intention and acting accordingly (Bagozzi \& Dholakia, 2006, p. 49).

Anticipated emotions differ from attitudes in their referents, underlying theoretical processes, and measurement (Bagozzi \& Dholakia, 2006, Perugini \& Bagozzi, 2001). First, attitudes in the TPB refer to action (i.e., attitudes toward the act), whereas anticipated emotions address anticipated goal attainment and goal failure following an action. Second, attitudes are passive reactions, and their effects require retrieval from memory of stored evaluations; attitudes reflect prior learning and function as learned dispositions. By contrast, anticipated emotions are dynamic in the sense of arising from thinking and appraisal processes at the time of decision making and involve forwardlooking judgments and feelings; they change as the contingencies and values of goals and their attainment change. Attitudes tend to be stable across situations, whereas the functioning of anticipated emotions is more situation-specific and therefore changeable. Third, attitudes are measured on bipolar semantic differential items; whereas anticipated emotions are measured by unipolar items, as is now the norm in the psychology literature. In sum, attitudes and anticipated emotions are measured and experienced differently and function differently in decision making. Measurements of both therefore should demonstrate discriminant validity, and both can have distinct effects on desires or intentions.

Desires and PB. Another limitation of the TPB is its failure to address how intentions become energized (Fazio, 1995, pp. 271-272): "The antecedents in the TPB provide rational reasons for acting ... but do not incorporate explicit motivational content needed to induce an intention to act" (Bagozzi \& Dholakia, 2006, p. 50). Bagozzi (1992) argues that desires provide the motivational impetus for intentions to act and suggests that attitude and other antecedents in decision-making work through desires to influence intentions. This happens through the operation of volitive desires or/and appetitive desires (Davis, 1984a,b). Volitive desires occur when reasons for acting are taken into account to form a self-commitment to act; once one is aware of and accepts his or her desire to act (i.e., is committed to act), this will motivate him or her to form an intention to act. Appetitive desires happen when reasons for acting serve as catalysts to release or free up a hidden or latent desire related to such biological needs as food, sex, or safety. To sum up, desires transform reasons for acting into self-regulated motives to act, which are the proximal causes of intentions. Therefore, the MGB includes desires in its formulation too as a mediator of the effects of attitudes and other antecedents and reasons to act on intentions.

A final difference between the TPB and the MGB is the inclusion of $\mathrm{PB}$ in the latter. Consistent with the theory of trying (Bagozzi \& Warshaw, 1990), the more often a series of acts has been performed, the more likely that this reflects successful decision making in the past and/or favorable evaluations of past actions and their implications, and the more likely one will continue to do so in the future. In this study, PB functions as a statistical proxy for habitual processes or omitted determinants of decisions based on prior learning, such that tests of hypotheses concerning the effects of attitudes, SN, PBC, and anticipated emotions on desire, and desire on intentions, are conducted by holding constant prior learning. Including PB in the model thus makes it more difficult to find significant effects for the other predictors. Further, prior research shows that the control of $\mathrm{PB}$ increases explained variance in intentions and reflects the influence of variables working through learned responses (Charng et al., 1988). When behavior has been practiced frequently in stable contexts, PB may influence future behavior directly, without impacting intention formation (Bagozzi \& 
Warshaw, 1990; Ouellette \& Wood, 1998). When PB has not been repeated often or when the context of performance changes or is difficult, $\mathrm{PB}$ has an indirect effect on future behavior through its impact on conscious intentions. In such cases, Ouellette and Wood (1998) argue, PB directly informs intentions for future responses through self-perception (Bem, 1972) and cognitive consistency (Festinger, 1957) processes; PB also potentially contributes to intentions by affecting PBC (Ajzen, 1991) and attitude (Eagly \& Chaiken, 1993).

\section{Social Identity}

A shortcoming of both the TPB and MGB is the neglect of social factors in consumption beyond compliance processes (i.e., beyond the effects of SN). Bacalhau dinners are usually jointly consumed with family members or friends, most often on holidays; in other words, bacalhau consumption typically takes place in a social setting. How can comprehensive social reasons for acting in such consumption contexts be better captured in a theoretical model? A need exists to account for social identification or internalization processes (e.g., Kelman, 1974). Eagly and Chaiken (1993, p. 683) note that "features embedded in certain types of role relationships," rather than compliance-targeted "stimuli or cues," need to be considered in studying the social context of action. In bacalhau consumption, compliance is not the only type of social influence that occurs. A different aspect of social influence that needs to be considered is the closeness and personalness of the social relations among persons who jointly consume the bacalhau dinner, if one wants to capture the mutual "caring" aspects of family meals.

How might the social relations one has with persons whom one prepares and/or consumes a bacalhau dinner be modeled? One way to address such social processes is to consider the construct of social identity. A person achieves a social identity through selfawareness of one's membership in a group and the emotional and evaluative significance of this membership (Tajfel, 1978). Researchers recently proposed three specific components for one's social identity: cognitive, evaluative, and emotional components (Bergami \& Bagozzi, 2000; Ellemers, Kortekass, \& Ouwerkerk, 1999). Social identity reflects a more group-based process than the circumscribed interpersonal-based dynamics of social compliance as captured by SN. When one prepares a bacalhau dinner for the immediate family and close relatives, he or she often has persons from such a core group in mind and envisions consumption as an extended social process involving these persons collectively. One's relationships and/or identification with these persons will have an impact on consumption decision-making. Summing up, to better capture the specific sociocultural context of the current study, the MGB is modified by introducing the construct of social identity into the model.

\section{The Moderating Effect of Cultural Orientation and Involvement with Food}

Bacalhau consumption is an important element in Portuguese culture. Bacalhau has been consumed in Portugal for over 500 years and is deeply embedded in Portuguese tradition and identity. Family social relationships in Portugal, compared to Northern Europe, tend to be more hierarchical and family-centered. However, even in southern Europe, people differ in the degree to which they exhibit independent versus interdependent self-construals (Markus \& Kitayama, 1991). Previous research claims that cultural orientation can be measured in four different dimensions: vertical/horizontal individualism and vertical/horizontal collectivism (Singelis, Triandis, Bhawuk, \& Gelfand, 1995). The country of our study, Portugal, is an individual culture, but is family-based. According to Triandis and Singelis (1998), Scandinavian countries can be described as horizontal individualistic cultures; but much of the rest of Europe can be best described as vertical individualistic cultures. Therefore, vertical individualism (VI) is argued to fit the research context better than horizontal individualism (HI), horizontal collectivism (HC), or vertical collectivism (VC). In sum, how vertical individualism (VI) influences consumption decisions is examined in the current study.

Furthermore, the preparation process for bacalhau dinners is complex and time consuming and requires special skill and knowledge. ${ }^{1}$ People with different levels of interest and involvement in food preparation in general should exhibit different levels of intentions toward bacalhau consumption. Previous studies (e.g., Xie, Bagozzi \& Troye 2008) conducted in Northern Europe show that people who are interested in food preparation in general are more likely to engage in specific, elaborate food consumption behaviors than people with lower levels of general interest. So, the impact of people's general interest in food preparation on their decisions toward bacalhau consumption is also tested.

The impact of cultural orientation and general interest in food preparation on bacalhau consumption will be tested by treating these two variables as moderating factors for the key relations specified in the MGB (and by implication also the TPB). It is predicted that the degree of influence amongst variables in tests of the TPB and MGB will be contingent on the level of vertical collectivism and the level of interest in food consumption.

\section{THEORY BUILDING AND THEORY TESTING}

In this section, the utility of the proposed theory is shown by progressively testing the TPB and increasingly complex versions of the MGB that capture the

\footnotetext{
1 First, the dry flat fish is personally selected and cut to order in the shop. Then, in the home, the fish is desalted in water for up to 72 hours, often in multiple soakings, before it can be prepared and cooked in particular classical culinary ways and served.
} 
different layers of evaluative, emotional, social, and cultural factors in the bacalhau consumption process. First, the evaluative process involved in bacalhau consumption is tested under the original TPB as a baseline. Based on the interpretation of the empirical results, a higher order construct is then introduced into the original model to capture the organization of deliberative processes antecedent to intentions to act (see Hagger $\&$ Chatzisarantis, 2005 for a similar approach). The revised TPB is compared with the original one. Second, the MGB is tested, which extends and deepens the TPB and is able to account for the emotional processes in bacalhau consumption. The MGB is tested and compared with the revised TPB. Then, the MGB is modified by introducing the construct of social identity to capture social process not encompassed by SN. Finally, the possible influence of the general cultural context and the effect of people's involvement with food consumption are examined. These two factors function to contingently regulate decision making.

\section{Method}

Participants and Procedures. Because it is most common for women to prepare bacalhau meals in Portugal, 153 female participants were recruited for this survey by a professional recruiting company, where data collection took place in Porto, Portugal. The participants were recruited from a consumer panel; they consumed bacalhau about at the national average. ${ }^{2}$

The data collection for the current study was part of a larger study, which involved testing and evaluating bacalhau products and then answering questionnaires about bacalhau consumption. To facilitate data collection, the pre-recruited participants were organized in groups of 10 . Each data collection session with 10 people lasted about 90 minutes. A total of 22 sessions was conducted over three days. This procedure has been used successfully by other researchers in previous studies (e.g., Sogn-Grundvåg \& Østli, 2009).

Sample characteristics of this group were as follows. Ages of respondents ranged from 22 to 68 (mean = $42.69, \mathrm{SD}=11.74)$, the majority $(96 \%)$ had a household size from one to four additional members, and household income ranged from 6000 to 36,000 euros per year. The measures used in this study follow common practice and were based on items in the literature for investigation of the TPB, MGB, and related research, and are available from the authors on request.

Data Analysis. CFA and structural equation models were used to test the different models in our study. The LISREL program was employed (Jöreskog \& Sörbom 2003), and four goodness-of-fit indices were applied to assess the fits of the estimated models: $\chi^{2}$-tests, Root

2 Average consumption level of bacalhau in Portugal is about one meal per person per week.
Mean Square Error of Approximation (RMSEA), Comparative Fit Index (CFI), and Non-Normed Fit Index (NNFI). Satisfactory model fits are indicated by nonsignificant $\chi^{2}$-tests, RMSEA values less than or equal to 0.07 , and CFI and NNFI values greater than or equal to 0.95 (Hu \& Bentler, 1999). Two indicators were used to operationalize each latent variable in the TPB. All analyses were performed on covariance matrices.

\section{Results}

\section{Part 1: Testing the Original TPB}

Evaluative processes are examined by applying the TPB as a baseline model. Given the likelihood that the TPB is too simplified to fully explain such complex food consumption behaviors as preparation of bacalhau dinners, the testing will be enlarged and adjusted in steps to accommodate the complexity of bacalhau consumption behavior. Further, the explanatory mechanisms required for this consumption behavior are compared with that needed to account for a single action of an individual consumer.

The means, standard deviations, and reliabilities for the scales are presented in Table 1. According to Nunnally (1978) values of 0.60 or larger are sufficient for reliability in explorative studies using such methods of analysis as multiple regression. In measures applied in this study, only SN and PBC have reliability under 0.60 . Both constructs were measured by two items; so, correlation of the two items was reported instead for reliability. SN has a reliability of 0.55 and $\mathrm{PBC} 0.56$, somewhat below 0.60 . However, for conducting tests by use of structural equation models, where measurement error and hence reliability is taken into account explicitly, it is permissible to have some constructs operationalized with two items correlated at the levels found herein.

Convergent and discriminant validity are ensured by checking that the CFA model fit well, factor loadings were high, and factors were correlated at levels significantly less than 1.00 . The results are presented in the path model for the TPB is shown in Figure 1.

Table 1. Means, Standard Deviations, and Reliabilities of Scales.

\begin{tabular}{|c|c|c|c|}
\hline Scale & Mean & $\begin{array}{l}\text { Standard } \\
\text { Deviation }\end{array}$ & Reliability \\
\hline Attitudes & 6.34 & 0.83 & 0.77 \\
\hline Subjective norms & 5.98 & 0.85 & 0.55 \\
\hline Perceived behavioral control & 6.12 & 0.84 & 0.56 \\
\hline Intentions & 6.09 & 0.95 & 0.77 \\
\hline Positive anticipated emotion & 5.64 & 0.79 & 0.70 \\
\hline Negative anticipated emotion & 3.95 & 1.38 & 0.69 \\
\hline Past behavior & 4.40 & 1.66 & 0.72 \\
\hline Desires & 5.42 & 1.03 & 0.79 \\
\hline Social Identity & 6.34 & 0.62 & 0.79 \\
\hline General food involvement & 5.52 & 1.42 & 0.81 \\
\hline Vertical Individualism & 3.78 & 1.26 & 0.73 \\
\hline
\end{tabular}




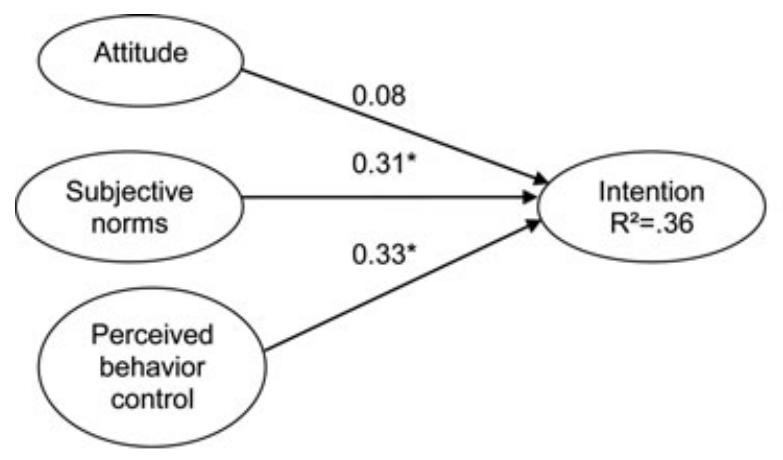

Chi-square $(14)=15.32$. RMSEA $=0,007 . N N F I=1,00 ., C F I=1,00$

Figure 1. The theory of planned behavior (original TPB).

Table 2 presents the results for correlations of factors as derived from a CFA. The model for the TPB fits very well: $\chi^{2}(14)=15.32, p=0.36$, RMSEA $=0.007$, $\mathrm{NNFI}=1.00$, and $\mathrm{CFI}=1.00$.

As hypothesized, intention to prepare a bacalhau dinner is significantly influenced by $\mathrm{SN}$ ( $\beta=0.31$, $t=3.09, p<0.01)$ and $\operatorname{PBC}(\beta=0.33, t=2.55, p<0.05)$. The explained variance in intentions is 0.36 . SN and PBC had equally strong influences on intention to prepare a bacalhau dinner. However, attitudes were found to be nonsignificant predictors of intention $(\beta=0.08$, n.s.). A closer inspection of the findings revealed that attitudes correlate moderately highly with intention $(r=0.37)$ and highly with PBC $(r=0.51)$. Therefore, it is likely that the nonsignificant path from attitudes to intention is due to multicolinearity. One way to overcome drawbacks with multicolinearity in structural equation models is to construct a secondorder factor for the highly correlated exogenous latent variables (e.g., Hagger \& Chatzisarantis, 2005). This remedy is only appropriate when the proposed firstorder factors can be considered dimensions or components of a single, higher order construct at a higher level of abstraction. In this case, attitudes, SN, and PBC can be seen as distinct, but highly correlated, aspects of a higher order deliberative process entailed under the TPB. They are distinct at a first-order, relatively concrete level of abstraction, but they also share significant common variance at a more abstract level, since they are highly and uniformly correlated. It can be hypothesized that a common source of variance underlies behavior of the first-order evaluative constructs, such that the evaluations can be considered special cases or subcomponents of a singular underlying psychological process at the highest level of abstraction. The highest level is termed, deliberative processes, but each component at the lowest level is a different, yet highly correlated, evaluative reaction on the part of consumers. Consequently, a second-order deliberative processes factor was introduced with the three latent variables-attitudes, $\mathrm{SN}$, and $\mathrm{PBC}$-loading on this second-order factor, and with deliberative processes, in turn, directly influencing intentions, in the analytical model. Such use of second-order factors is becoming common in psychology (e.g., Hagger \& Chatzisarantis, 2005), and marketing (e.g., Bagozzi \& Dholakia, 2006) in recent years.

\section{Testing the Revised TPB}

Figure 2 presents the results for the revised model of the TPB. This model fits very well: $\chi^{2}(16)=17.76, p=$ 0.34 , RMSEA $=0.015$, NNFI $=1.00$, and CFI $=1.00$. Attitudes, SN, and PBC all loaded highly on the secondorder deliberative processes factor. These loadings provide an indication of the relative contributions of the components to the second-order factor, where $\mathrm{PBC}$ is the strongest component ( $\beta=0.78, t=4.59, p<0.001$ ), $\mathrm{SN}$ is slightly less strong ( $\beta=0.68, t=3.58, p<0.001$ ), and attitudes slightly lower in turn $(\beta=0.61, t=5.24$, $p<0.001$ ). Yet all three components are strongly related to the deliberative processes factor. Deliberative processes significantly influence intentions to prepare a bacalhau dinner $(\beta=0.69, p<0.001)$. The explained variance in intentions increased to 0.48 , over that found for the traditional TPB (i.e., 0.36).

The results show that attitudes, SN, and PBC can be conceived as components of a more abstract summary construct termed, deliberative processes. Attitudes refer to evaluative reactions toward an action and are thought to reflect predispositions to respond in a

Table 2. Correlation Matrix of Latent Constructs.

\begin{tabular}{lcccccccccr}
\hline & PB & A & SN & PBC & PE & NE & Desire & SI & Intention & GDP \\
\hline PB & 1.00 & & & & & & & & & \\
A & 0.30 & 1.00 & & & & & & & \\
SN & 0.29 & 0.42 & 1.00 & & & & & & \\
PBC & 0.35 & 0.51 & 0.49 & 1.00 & & & & & \\
PE & 0.07 & 0.27 & 0.26 & 0.32 & 1.00 & & & & \\
NE & 0.04 & 0.17 & 0.17 & 0.20 & 0.45 & 1.00 & & & & \\
Desire & 0.47 & 0.47 & 0.46 & 0.56 & 0.48 & 0.30 & 1.00 & & & \\
SI & 0.15 & 0.29 & 0.28 & 0.34 & 0.19 & 0.12 & 0.41 & 1.00 & & \\
Intention & 0.37 & 0.37 & 0.36 & 0.44 & 0.38 & 0.24 & 0.79 & 0.33 & 1.00 & 1.00 \\
GDP & 0.45 & 0.66 & 0.63 & 0.77 & 0.42 & 0.26 & 0.72 & 0.44 & 0.57 & 1.00 \\
GE & 0.08 & 0.33 & 0.31 & 0.38 & 0.84 & 0.53 & 0.57 & 0.22 & 0.45 & 0.50 \\
\hline
\end{tabular}

Note: $\mathrm{PB}=$ past behavior; $\mathrm{A}=$ attitude; $\mathrm{SN}=$ social norms; $\mathrm{PBC}=$ perceived behavioral control; $\mathrm{PE}=$ anticipated positive emotions; $\mathrm{NE}=$ anticipated negative emotions; $\mathrm{SI}=$ social identity; GDP = global deliberative processes; GE = global emotions. 


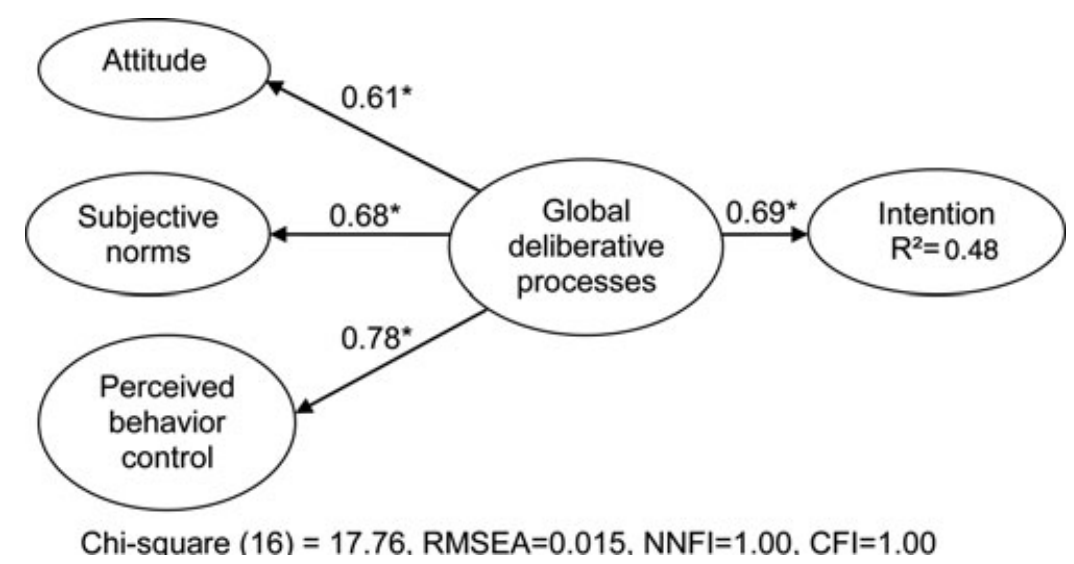

Figure 2. The theory of planned behavior with a second-order factor (revised TPB; parameters significant at $p$-levels of 0.05 or better are marked with asterisks).

favorable or unfavorable manner (Eagly \& Chaiken 1993); SN capture felt social pressure and reflect a need for approval; PBC is one's judgment that he or she can perform an action and account for behavior not fully under volitional control (Ajzen, 1991). When emotional processes and habitual processes are introduced below, this interpretation of the second-order factor as a presentation of global deliberative processes (GDP) will become clearer and its broader utility demonstrated.

To sum up, the strength of the TPB is its simplicity and its applicability to a wide range of social behaviors. However, the TPB fails to capture emotional content and noncompliance-related social content such as social identity, which is based on identification processes. These processes are considered hereafter.

\section{Part 2: Testing the Original MGB}

A similar procedure was applied to test the MGB as was done with the TPB. Two indicators were used to operationalize each latent variable. For latent variables with more than two items, items were combined to produce two indicators, using the so-called partial disaggregation model recommended in the literature (Bagozzi \& Heatherton, 1994). For instance, odd-numbered measures of positive anticipated emotions were averaged to form the first indicator, and the remaining even numbered items were used to form the second indicator. This approach yields models with less parameter to estimate and a reasonable ratio of cases to parameters to facilitate estimation, while smoothing out measurement error to a certain extent. It should be used only when factor analyses of items prove that all measures load on one factor and thus can be considered parallel measures of the factor and parceled, as done in this study. The means, standard deviations, and reliabilities for the scales used for the MGB are summarized in Table 1. For assessing convergent and discriminant validity, the CFA model fit well, factor loading were high, and the correlations among the latent constructs were significantly less than one.
The results for the test of the MGB can be seen in Figure 3. This model fits very well: $\chi^{2}(90)=124.82$, RMSEA $=0.048$, NNFI $=0.97$, and CFI $=0.98$. Similarly, attitudes, SN, and PBC all loaded at high levels on a second-order GDP factor. The loadings show that $\mathrm{PBC}$ is the strongest component $(\beta=0.75, p<0.001)$, while $\mathrm{SN}(\beta=0.66, p<0.001)$ and attitudes $(\beta=0.67$, $p<0.001)$ are slightly less strong. Desire to prepare a bacalhau dinner was influenced significantly by both $\operatorname{GDP}(\beta=0.61, p<0.01)$ and negative anticipated emotions $(\beta=0.28, p<0.01)$. However, $\mathrm{PB}(\beta=0.15$, n.s. $)$ and positive anticipated emotions $(\beta=0.03$, n.s. $)$ did not influence desires. Sixty-five percent of the variance in desire was explained. Desire had a strong influence on intentions ( $\beta=0.79, p<0.001)$, with $63 \%$ of variance explained in intentions; this can be compared to $48 \%$ explained variance in intentions under the revised TPB and $36 \%$ under the original TPB.

The results show that the MGB provides a fuller explanation of decision making than that provided by the TPB. Not only is significantly more variance explained under the MGB versus the TPB, which is an empirical advantage, but conceptually the MGB provides a richer picture of how decisions are made compared to the TPB. Under the MGB, reasons for acting are integrated and resolved by desires, before going on to influence intentions. These reasons not only include the classic deliberative determinants found under the TPB, but they also encompass emotion in the form of anticipated affect related to the consequences of acting and $\mathrm{PB}$. The MGB thus constitutes a richer explanation of decision making than the TPB.

Anticipated negative emotions significantly influenced desires, as hypothesized (see Figure 3). But anticipated positive emotions surprisingly was not a significant predictor, yet it was moderately highly correlated with desire $(r=0.48)$ in the raw data. Because anticipated positive emotions correlate highly with anticipated negative emotions $(r=0.45)$, it is possible that the nonsignificant path from anticipated positive emotions to desire is due to multicolinearity. By constructing a second-order factor for anticipated 


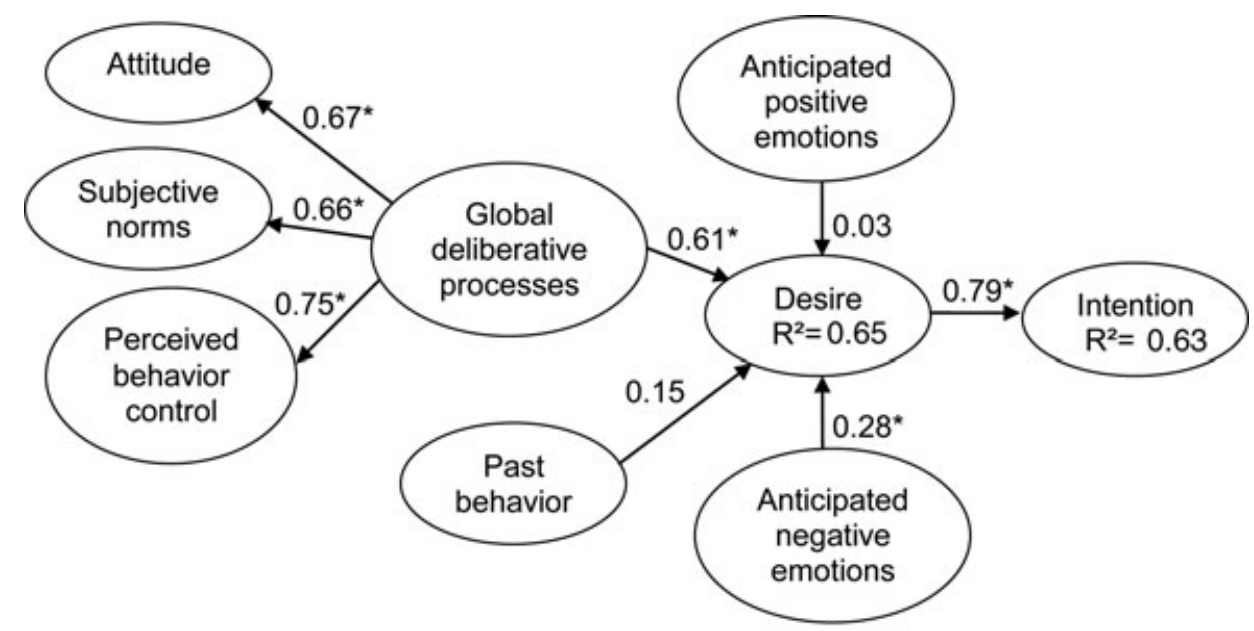

Chi-square $(90)=124.82$. RMSEA $=0.048 . \mathrm{NNFI}=0.97 . \mathrm{CFI}=0.98$

Figure 3. The model of goal-directed behavior with one second-order factor for deliberative processes. (parameters significant at $p$-levels of 0.05 or better are marked with asterisks).

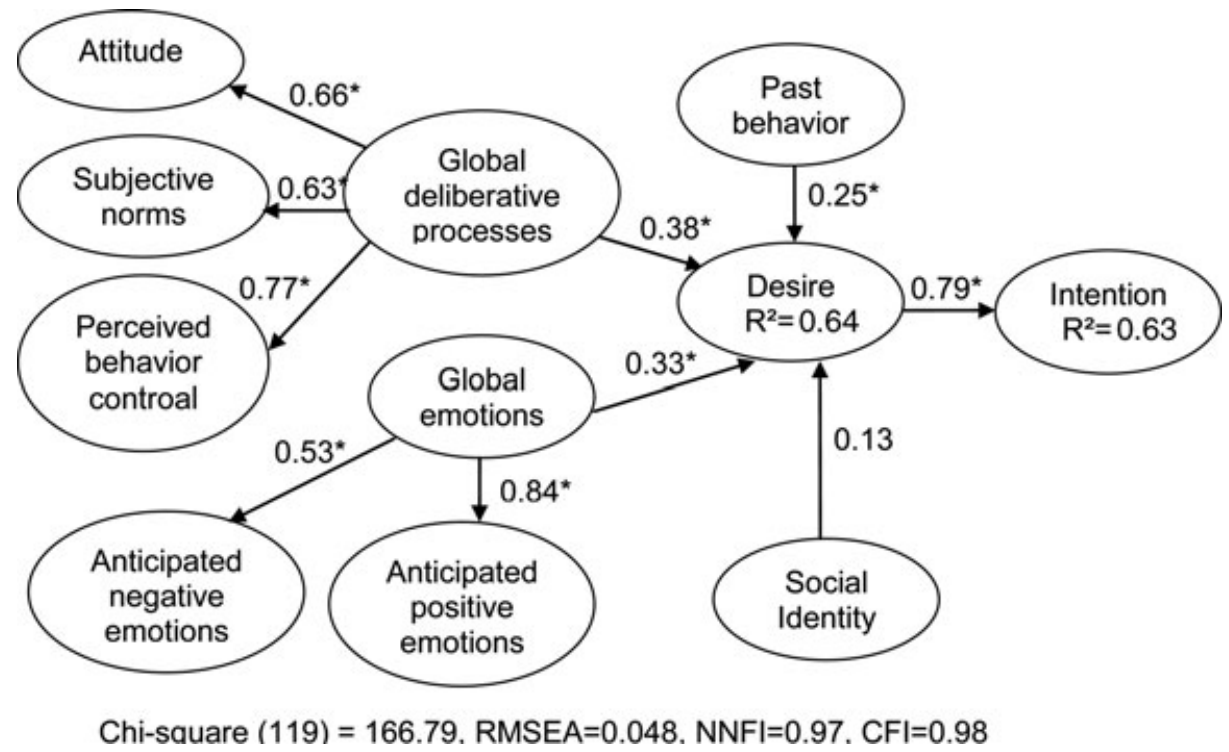

Figure 4. The model of goal-directed behavior with social identity and one second-order factor for deliberative processes and one second-order factor for emotions (parameters significant at $p$-levels of 0.05 or better are marked with asterisks).

positive and negative emotions, one may be able to capture the independent contributions of each and overcome multicollinearity problems. In the next step, the MGB is modified to investigate this possibility. Furthermore, the construct of social identity is introduced to capture social processes other than compliance process encompassed by SN.

\section{Testing a Modified MGB by Introducing Social Identity and Representing Global Emotions}

A similar procedure is applied to test the modified MGB as in the past section of the paper. The findings for the path model are presented in Figure 4. This model fits very well: $\chi^{2}(119)=166.79, \mathrm{RMSEA}=0.048, \mathrm{NNFI}=$ 0.97, and $\mathrm{CFI}=0.98$. Attitudes, $\mathrm{SN}$ and $\mathrm{PBC}$ again all loaded at high levels on the second-order GDP factor. For the other second-order global anticipated emotions factor, both anticipated positive emotions $(\gamma=0.84)$ and negative emotions ( $\gamma=0.53)$ loaded highly. Importantly, compared to our test of the original MGB shown in Figure 3, the revised MGB displayed in Figure 4 demonstrates that desire to prepare a bacalhau dinner is significantly influenced by $\operatorname{GDP}(\beta=0.38, t=2.61$, $p<0.01)$ and global anticipated emotions $(\beta=0.33, t=$ $2.38, p<0.05)$. PB $(\beta=0.25, t=2.73, p<0.01)$ also had a significant impact on desire. Social identity, however, failed to significantly predict desire $(\beta=0.13, t=1.65$, 
n.s.). Sixty-four percent of the variance in desire was explained. Desire had a strong influence on intentions $(\beta=0.79, t=11.47, p<0.001)$. The explained variance in intentions is 0.63 .

The results show that both GDP and global anticipated emotions strongly influence the desire to prepare a bacalhau dinner. It appears that both rational and emotional processes are important in people's decision making for bacalhau consumption; this conclusion fits well with everyday observations of the Southern European cultural context of the current study. In addition, habitual processes contribute unique variance over and above the reasoned and emotional forces. However, social identity failed to significantly predict desire. Possible reasons for this finding will be offered in General Discussion section.

\section{Tests of Mediation for the Revised MGB}

A formal test of mediation implied by the revised MGB was also conducted by following a procedure analogous to that proposed by Baron and Kenney (1986) but using the more powerful structural equation methodology. The model pictured in Figure 4 serves as a baseline for $\chi^{2}$-difference tests of direct paths from antecedents of desires to intentions. The results show that direct paths from $\mathrm{PB}$ and social identity to intentions were not significant, so desire fully mediates the effects of PB on intentions, but social identity fails to influence intentions either indirectly through desire or directly. The second-order factor of GDP has a positive and significant effect on intentions, so desire partially mediates the effects of GDP on intentions.

Surprisingly, the second-order factor of global emotions (GE) has a significant and negative effect on intentions. It is possible that the significant, negative path from GE to intentions is also due to multicolinearity because of the high correlation of anticipated emotions with desire $(\gamma=0.66)$. A third-order factor for GDP and global anticipated emotions was conducted to overcome such multicolinearity. Both second-order factors for GDP and global anticipated emotions loaded highly on the third-order factor, which in turn influenced desire strongly. This confirmed the initial suspicion that the significant negative path from GE to intentions was caused by multicolinearity.

\section{Part 3: Testing Moderating Effects of Cultural Orientation and Involvement with Food}

Finally, moderating effects on decision making of two processes are tested. These processes are particularly relevant to the present study of bacalhau consumption: cultural orientation and involvement with food. This is done by examining whether the key relations specified in the modified MGB (and by implication the TPB) function in contingent senses. Cultural orientation,
VI (vertical individualism) in the present case, was measured by a four-item subscale from Singelis et al.'s (1995) individualism-collectivism scale, whereas involvement with food was measured by the Food Involvement Scale developed by Bell and Marshall (2003) and Marshall and Bell (2004). Limited by relatively small sample size of the current study, it is not possible to test moderating effects with LISREL, so multiple regression analysis is applied instead (e.g., Aiken \& West, 2003; Jaccard \& Turrisi, 2003). All variables were mean centered before conducting the regression analyses. The results are summarized below.

\section{The Moderating Effect of Vertical Individualism}

The results show that vertical individualism (VI) moderates the effects of antecedents on desires. Vertical individualism positively moderates the effects of attitudes $(\beta=0.17, t=2.81, p<0.01)$, PBC $(\beta=0.13$, $t=1.92, p<0.06)$, and negative anticipated emotions on desire $(\beta=0.10, t=2.09, p<0.05)$. Vertical individualism captures the cultural value of doing better than others. As shown in Figure 5, for those who are low in VI (e.g., those who are less competitively oriented), their attitudes, $\mathrm{PBC}$, and negative anticipated emotions have no effects on desire; for those high in VI, the greater their attitudes, $\mathrm{PBC}$, and negative anticipated emotions, the stronger are their desires to prepare bacalhau meals for the family.

\section{The Moderating Effect of Involvement with Food}

The findings show that involvement with food moderates the effects of $\mathrm{PB}$, attitudes, and negative anticipated emotions on desire to prepare a bacalhau dinner. As shown in Figure 5, involvement with food negatively moderates the effect of $\mathrm{PB}$ on desire $(\beta=-0.11, t=$ $-3.40, p<0.001)$. This implies that, for those with low general involvement in food preparation, their desire to prepare a bacalhau dinner will be strongly influenced by past experiences with bacalhau preparation. For those low in involvement, the more often and more recently they prepared a bacalhau dinner, the stronger their desire to do so again. However, those highly involved in food preparation in general are more likely to have a strong desire to prepare a bacalhau dinner to begin with, no matter what the extent of past experience is.

Moreover, involvement with food negatively moderates the effect of attitudes $(\beta=-0.13, t=-1.94, p<$ 0.06 ) on desire. As shown in Figure 6, for those with low general involvement in food preparation, the more positive their attitudes, the stronger their desire to prepare a bacalhau dinner. But for those who are high in general food involvement, their desire to prepare a 


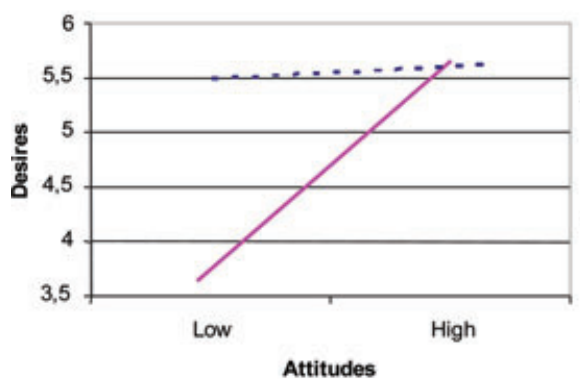

Moderating effect of vertical individualism

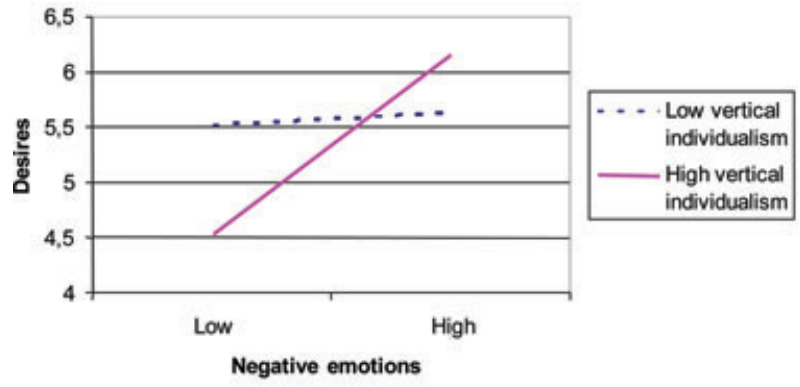

Moderating effect of Vertical Individualism

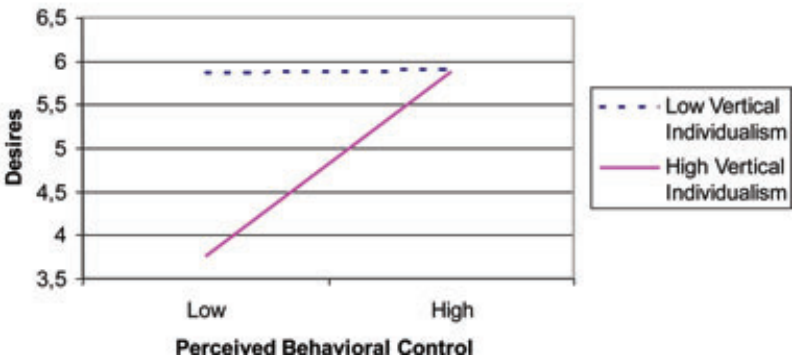

Figure 5. Moderating effect of vertical individualism on effects of attitudes, negative anticipated emotions, and perceived behavioral control on desires.

bacalhau dinner is strong, no matter what their attitudes are.

Finally, a negative moderating effect of involvement with food was found on the influence of negative emotions on intention $(\beta=-0.09, t=-2.41$, $p<0.05)$. For those with low involvement in general food preparation, their desires to prepare a bacalhau dinner are more likely to be influenced by their anticipated negative emotions than those who are highly involved (see Figure 6). The greater the negative anticipated negative emotions, the stronger the desire to prepare a bacalhau dinner, for those low in general food involvement. For those high in food involvement, negative anticipated emotions have no effect.

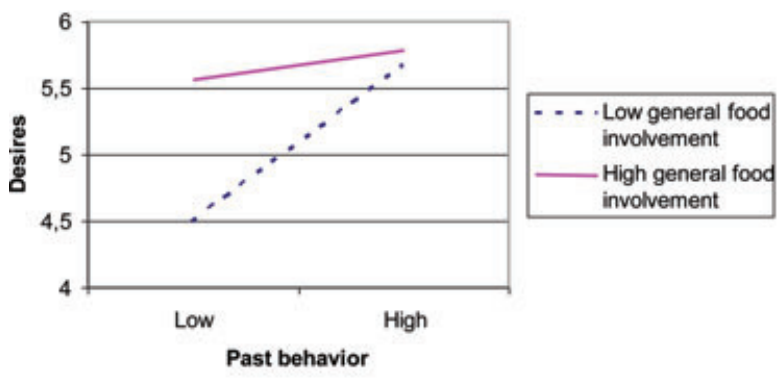

Moderating effect of general food involvement

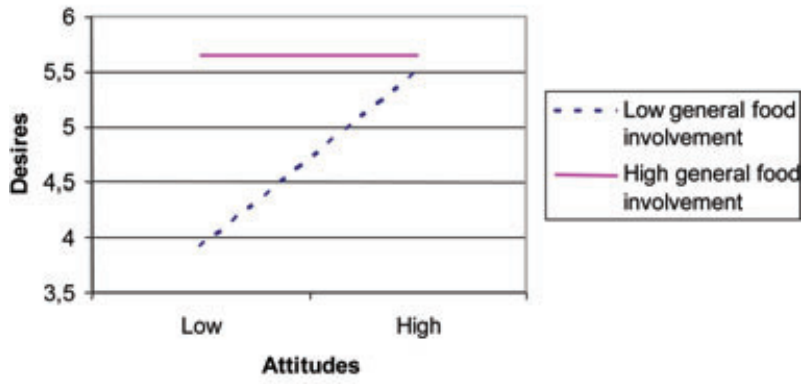

Moderating effect of general food involvement

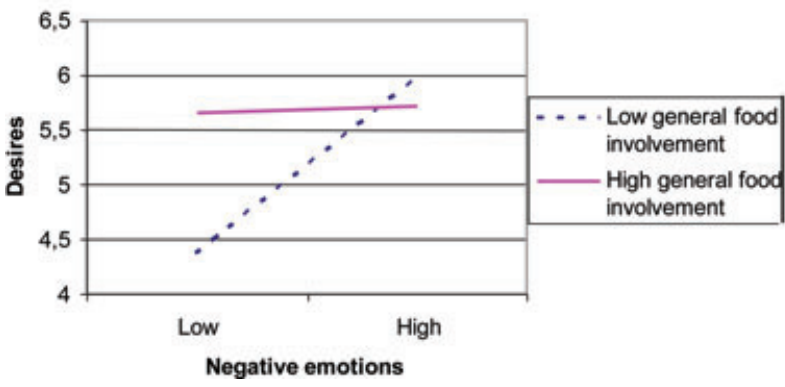

Figure 6. Moderating effect of general food involvement on effects of past behavior, attitudes, and negative anticipated emotions on desires.

\section{GENERAL DISCUSSION}

\section{Deliberative Processes Accounted for by TPB}

The explanation of some consumer behaviors, in this study bacalhau consumption decisions in Portugal represents complex challenges for extant theories of decision making. The application of the TPB provided only a partial explanation of bacalhau consumption decisions. This was perhaps to be expected because of the multifaceted social and emotional nature of bacalhau 
consumption in Portugal. The TPB appears to be less useful for cases of behavior where decisions encompass planning and multiple stages and where emotional feelings and cultural concerns are infused in the process. Bacalhau purchase, preparation, and fulfillment are done with the family and traditional customs in mind. Bacalhau consumption decisions, even when viewed through the eyes of a prime mover in the process, entail a holistic melding together of psychological, social, and cultural anticipation, unlike many behaviors that might be reduced to the actions of an individual in more or less isolation, such as the purchase of low cost, frequently purchased essentials (e.g., tooth paste, soap, breakfast cereal, bread).

Nevertheless, the TPB still constitutes part of the framework developed herein. It was discovered that reasoned expectations with respect to consequences of acting-in terms of attitudinal, subjective normative, and perceived control factors-were essential determinants of decision making. Yet even here, it necessary to adjust the TPB to better accommodate the large amount of common variance shared by the three classic determinants of decision making: attitudes, SN, and PBC. Rather than functioning as three distinct, independent causes of intentions, the classic antecedents of decision making formed three facets of a higher order, GDP construct.

Decision makers in the current study of bacalhau consumption experienced a general overall sense of rational reasons for acting, rather than distinct logical or sensible criteria operating distinctly. This is a new way of modeling the determinants of reasoned action, and it is possible that other consumption contexts fit this reconceptualization well, too. It is speculated that some of past applications of the TPB that have had mixed success in terms of significance of individual predictors may have been the result of a failure to consider such higher order abstract representations as considered herein (cf., Bagozzi, Lee, \& Van Loo, 2001; Hagger $\&$ Chatzisarantis, 2005).

\section{Emotional and Social Processes Addressed by the Modified MGB}

Anticipated Emotions. To address a fundamental shortcoming in the TPB, at least for such sociocultural cases as bacalhau consumption, the MGB was applied. The MGB introduces three innovations into the explanation of consumer behavior, over and above the TPB. First, it gives a parsimonious means for incorporating emotional aspects of decision making. Positive and negative anticipated emotions, as a consequence of appraising expected future success and failure in consumption, capture the intimate feelings a decision maker has in relation to the performance and outcomes of consumption (e.g., Bagozzi, Baumgartner, \& Pieters, 1998). Second, the MGB adds PB as a predictor of decision making. This has the dual effect of accounting for ha- bitual processes and controlling for omitted variables related to prior learning. In so doing, it also provides for a tougher test of the effects of deliberative and emotional processes, because for these latter determinants to have effects, they must contribute unique content beyond any residual learning associated with them and captured by $\mathrm{PB}$ as a control in tests of hypotheses.

Desire. Finally, perhaps the most important additional contribution of the MGB concerns bringing in the key role of desire in decision making. A limitation of the TPB and similar approaches is that theory is silent on how the numerous determinants of intentions come together to influence decision making. These frameworks all posit antecedents as parallel, independent causes of decisions and leave open, or to empirical determination, how the antecedents function relatively in their impact, if any. By contrast, the MGB proposes that the antecedents to decision making are integrated and transformed functionally through the overall felt desire to act by a decision maker. Desire is hypothesized to reconcile the roles of various antecedents and to provide the proximal motivation to act. Intention supplies the commitment or volition to act, given a felt desire. The antecedents to decision making provide reasons for acting-rational, emotional, social-which stimulate the desire to act. Yet having a desire to act does not ensure that one will act, as one can desire something without intending to act on the desire. It is the influence of desire on intention that bridges mental processes and actual behavior needed to fulfill one's desires.

The findings for the MGB indeed demonstrated enhanced explanation over the TPB. This occurred empirically in the sense that explained variation increased from 0.36 under the classic TPB to 0.48 under the revised TPB and finally to 0.63 under the MGB. Conceptually, the MGB yields a more refined accounting of decision making by expanding antecedents to include emotion and $\mathrm{PB}$ and by providing a mechanism and motive converting multiple, disparate felt social psychological criteria into a decision to act or not. Desire is the cornerstone of this transformative process.

The MGB was next extended in two directions in the tests of hypotheses. First, the findings showed that high correlations between measures of positive and negative anticipated emotions induced multicollinearity, which in turn obscured the effects of positive anticipated emotions on desire. To overcome this, positive and negative anticipated emotions were proposed to be two components of a higher order global or overall anticipated emotion construct, and this construct was found to predict desire. The data indeed supported such a conjecture (see Figure 4). With this reformulation, the findings showed that desire influences intention and is in turn dependent on GDP and global anticipated emotions, as well as habitual processes captured by PB.

Social Identity. The second extension of the MGB was to include social identity as a predictor of desire, complementing the social effects of compliance that reside 
in SN. However, social identity failed to predict desire in tests of hypotheses. Why? Because measures of identity correlate highly and significantly with measures of desire and intention, it is possible that the most likely explanation is that the cognitive, affective, and evaluative aspects of social identity become absorbed into the deliberative and emotional content already in the MGB for the context at hand. Social identity is designed to accommodate group forces in decision making. But unlike recent applications of social identity in marketing, where small friendship groups were studied and it is the group that acts through its members (c.f., Bagozzi \& Dholakia, 2006), in this study it is not so much the family that acts as a unit as it is that individual family members act in a coordinated way to participate in a familiar occasion of family celebration. Indeed, because focus was on the primary person acting on behalf of the family in the execution of bacalhau consumption, it is likely that shared group feelings and attitudes become internalized into anticipated emotions and attitudes. Hence when social identity is added to the MGB in the context at hand, no additional unique variance exists to predict desire. Nevertheless, when a third-order factor was modeled with the GE and social identity loading on it, the factor had a significant effect on desire. Under these conditions, both positive and negative anticipated emotions and social identity contribute to influence desire. The third-order factor can be interpreted as the integration of felt emotions and social identity into a single construct, and this then permits one to look at their joint effects on desire. Thus, social identity affects intentions after all but at a high level of abstraction as a consequence of its functioning in its role as a part of a global summary of anticipated emotions and social identity in the family.

\section{The Impact of Cultural Orientation and Involvement with Food}

Finally, attention was paid to the possible impact of cultural nuances on decision making in bacalhau consumption. The current study was conducted in a southern European culture where family and social interactions are important. Moreover, individual differences exist across people based on degree of personal interest in food. Would people's cultural orientation and their involvement with food play a role in their decisionmaking process of bacalhau consumption? Hypotheses were tested by treating cultural orientation and involvement with food as two potential moderators of relationships in the MGB. The original MGB, like the TPB, makes hypotheses of linear effects and does not address possible interaction effects.

To measure involvement with food, a recent scale developed by Bell and Marshall (2003; Marshal \& Bell, 2004) was used to operationalize this personality trait. The findings showed that food involvement moderated the effects of $\mathrm{PB}$, attitudes, and negative anticipated emotions on desire to consume bacalhau. Significantly, these variables affected desire only for consumers low in food involvement; those high in food involvement already possessed strong desires to participate in bacalhau consumption. The second significant moderator, vertical individualism, is one of the four cultural orientation dimensions measured by Singelis et al.'s 16-item scale for individualism-collectivism (Singelis et al., 1995). Vertical individualism also regulated the effects of attitudes, negative anticipated emotions, and $\mathrm{PBC}$ on desires. For those high in vertical individualism, the aforementioned variables had positive effects on desires, whereas for those low in vertical individualism, their desires were unaffected by these variables and in fact were high under all conditions. The current research shows that the effects of key determinants of desire in the MGB are contingent on the traits of food involvement and degree of vertical individualism.

The two contingencies might have relevance for social policy and marketing. To get persons currently uninvolved with food to consume bacalhau meals, it seems possible to increase their desire for bacalhau by giving free samples or lessons in food preparation (thereby attempting to induce learning and work through PB effects in the future), targeting attitudes in advertisements or in communications made by fish mongers at the point of sale, and stressing negative emotional consequences (e.g., disappointment by family members) of not engaging in consumption of bacalhau meals. Likewise to get persons high in vertical individualism to increase their desire for bacalhau meals, it may be fruitful to target attitudes (again in persuasive communications), negative anticipated emotions (e.g., by emphasizing the loss of pride and family esteem that would occur if one failed to consume bacalhau), and PBC (by emphasizing ease of preparation, say).

\section{Empirical Context of This Study}

Finally, the empirical context of the current study has implications for other forms of consumption. First, in addition to Portugal, bacalhau is an important part of life in Brazil and is a common dish in Italy, Spain, Greece, and is occasionally eaten in Northern Europe, Africa, and the Caribbean as well. Second, food consumption of other foodstuffs in many parts of the world can be explained by the models developed and tested herein, to the extent that rational and emotional criteria, as well as social, cultural, and trait factors are important in decision making. Finally, the family social context of consumption investigated herein shows some similarity to decision-making families make with regard to recreation, travel, and purchases of everyday jointly consumed items.

\section{Limitation and Future Direction}

One limitation of the current study is that the sample size did not allow for multiple group analysis with structural equation modeling to test moderating effects 
of cultural orientation and specific interest with food. In future studies, larger sample sizes may be needed to explore the possible impact of cultural orientation and other moderators with SEMs. However, significant statistical interactions were found in this study, so the relatively small sample size was not a problem.

Another limitation of this study is that social identity failed to predict desire and intention in bacalhau consumption. As discussed earlier, it is most likely that the cognitive, affective, and evaluative aspects of social identity become absorbed into the deliberative and emotional content already in the MGB. Thus, future research should investigate consumption in family contexts where explicit sharing of goals and actions occur among family members and the measures of social identity are phrased so as to represent the joint processes. The tests of a third-order causal model lend support to the interpretation herein, as social identity in fact had indirect effects on desire.

Finally, the current study investigated quantitatively the emotional and social process of bacalhau consumption by theory building and theory testing based on extant models and proposed modifications. A promising future direction could be to conduct qualitative studies on bacalhau consumption and consumption of similar social-cultural-based goods to get richer insight into the deliberative, emotional, social, and cultural processes embedded in such complex consumption behaviors with the hope that new processes might be uncovered that could be incorporated in theory and development and future surveys.

\section{REFERENCES}

Aiken, L. S., \& West, S. G. (2003). Multiple regression: Testing and interpreting interactions. Newbury Park: Sage.

Ajzen, I. (1991). The theory of planned behavior. Organizational Behavior and Human Decision Processes, 50, 179211. doi:10.1016/0749-5978(91)90020-T

Armitage, C. J., \& Conner, M. (2001). Efficacy of the theory of planned behavior: A meta-analytic review. British Journal of Social Psychology, 40, 471-499. doi:10.1348/01446660 1164939

Astøsm, A. N., \& Rise, J. (2001). Young adults' intention to eat healthy food: Extending the theory of planned behavior. Psychology \& Health, 16, 223-237. doi:10.1080/08870440108405501

Bagozzi, R. P. (1992). The self-regulation of attitudes, intentions, and behavior. Social Psychology Quarterly, 55, 178204.

Bagozzi, R. P., \& Dholakia, U. M. (2006). Antecedents and purchase consequences of customer participation in small group brand communities. International Journal of Research in Marketing, 23, 45-61. doi:10.1016/j.ijresmar. 2006.01.005

Bagozzi, R. P., \& Heatherton, T. F. (1994). A general approach to representing multifaceted personality constructs: Application to state self-esteem. Structural Equation Modeling, 11, 35-67. doi:10.1080/10705519409539961

Bagozzi, R. P., \& Nataraajan, R. (2000). The year 2000: Looking forward. Psychology \& Marketing, 17, 1-11.
Bagozzi, R. P., \& Warshaw, P. R. (1990). Trying to consume. Journal of Consumer Research, 17, 127-140.

Bagozzi, R. P., Baumgartner, H., \& Pieters, R. (1998). Goal-directed emotions. Cognition and Emotion, 12, 1-26. doi:10.1080/026999398379754

Bagozzi, R. P., Lee, K.-H., \& Van Loo, M. F. (2001). Decisions to donate bone marrow: The role of attitudes and subjective norms across cultures. Psychology and Health, 16, 29-56.

Baron, R. M., \& Kenny, D. A. (1986). The moderator-mediator variable distinction in social psychological research: Conceptual, strategic, and statistical considerations. Journal of personality and social psychology, 51, 1173-1182. doi:10.1037/0022-3514.51.6.1173

Bell, R., \& Marshall, D. W. (2003). The construct of food involvement in behavioral research: Scale development and validation. Appetite, 40, 235-244. doi:10.1016/S01956663(03)00009-6

Bem, D. J. (1972). Self-perception theory. In L. Berkowitz (Eds.), Advances in experimental social psychology (Vol. 6, pp. 1-62). San Diego, CA: Academic Press.

Bergami, M., \& Bagozzi, R. P. (2000). Self-categorization and commitment as distinct aspects of social identity in the organization: Conceptualization, measurement, and relation to antecedents and consequences. British Journal of Social Psychology, 39, 555-577. doi:10.13 48/014466600164633

Charng, H-W., Pilavin, J. A., \& Callero, P. L. (1988). Role identity and reasoned action in the prediction of repeated behavior. Social Psychology Quarterly, 51, 303-317.

Davis, W. A. (1984a). A causal theory of intending. American Philosophical Quarterly, 21, 43-54.

Davis, W. A. (1984b). The two senses of desire. Philosophical Studies, 45, 181-195. doi:10.1007/BF00372477

Dennison, C. M., \& Sheppard, R. (1995). Adolescent food choice: An application of the theory of planned behavior. Journal of Human Nutrition and Dietetics, 8, 9-23. doi:10.1111/j.13 65-277X.1995.tb00292.x

Eagly, A. H., \& Chaiken, S. (1993). The psychology of attitude. Orlando, FL: Harcourt Brace Jovanovich College Publishers.

Ellemers, N., Kortekaas, P., \& Ouwerkerk, J. W. (1999). Self-categorisation, commitment to the group and group self-esteem as related but distinct aspects of social identity. European Journal of Social Psychology, 29, 371-389. doi:10.1002/(SICI)1099-0992(199903/05)29: 2/3<371::AID-EJSP932>3.0.CO;2-U

Fazio, R. H. (1995). Attitudes as object-evaluation associations: Determinants, consequences, and correlates of attitude accessibility. In R. E. Petty \& J. A. Krosnik (Eds.) Attitude strength: Antecedents and consequences (pp. 247282). Hillsdale, NJ: Lawrence Erlbaum Associates.

Festinger, L. (1957). A theory of cognitive dissonance. Evanston, IL: Row, Peterson and company.

Hagger, M. S., \& Chatzisarantis, N. L. D. (2005). First- and higher-order models of attitudes, normative influence, and perceived behavioral control in the theory of planned behavior. British Journal of Social Psychology, 44, 513-535. doi:10.1348/014466604x16219

Hu, L.-t., \& Bentler, P. M. (1999). Cutoff criteria for fit indexes in covariance structure analysis: Conventional criteria versus new alternatives. Structural Equation Modeling, 6, 1-55. doi:10.1080/10705519909540118

Jaccard, J., \& Turrisi, R. (2003). Interaction effects in multiple regression, 2nd ed. Thousand Oaks, CA: Sage.

Jöreskog, K. G., \& Sörbom, D. (2003). LISREL. Skokie, IL: Scientific Software International, Inc. 
Kelman, H. C. (1974). Further thoughts on the processes of compliance, identification, and internalization. In J. T. Tedeschi (Eds.). Social power and political influence (pp. 125-171). Chicago, IL: Aldine.

Leone, L., Perugini, M., \& Ercolani, A. P. (2004). Studying, practicing, and mastering: A test of the model of goal-directed behavior (MGB) in the software learning domain. Journal of Applied Social Psychology, 34, 1945-1973. doi:10.1111/j.1559-1816.2004.tb02594.x

Markus, H. R., \& Kitayama, S. (1991). Culture and self: Implications for cognition, emotion, and motivation. Psychological Review, 98, 224-253, doi:10.103710033-295x.98.2.224

Marshall, D. W., \& Bell, B. (2004). Relating the food involvement scale to demographic variables, food choice and other constructs. Food quality and preference, 15, 871-879. doi:10.1016/j.foodqual.2004.06.003

Nunnally, J. C. (1978). Psychometric theory (2nd ed.). New York, NY: McGraw-Hill.

Østli, J., Heide, M., Carlehög, M., \& Eilertsen, G. (2006). The importance of bacalhau consumption in Portugal and a preliminary product consumer test in Lisboa. In J. B. Luten, et al. (Eds.) Seafood research from fish to dish: Quality, safety and processing of wild and farmed fish (pp. 241-249). Retrieved from http:http:////www.cabdirect.org/ abstracts/20073061880.html;jsessionid $=533363 \mathrm{C} 2 \mathrm{CD} 04$ 4E81AD1B7FE96410FE2C.

Ouellette, J. A., \& Wood, W. (1998). Habit and intention in everyday life: The multiple process by which past behavior predicts future behavior. Psychological Bulletin, 124, 5474. doi:10.1037/0033-2909.124.1.54

Perugini, M., \& Bagozzi, R. P. (2001). The role of desires and anticipated emotions in goal-directed behavior: Broadening and deepening the theory of planned behavior. British Journal of Social Psychology, 40, 79-98. doi:10.1348/014466601164704

Perugini, M., \& Conner, M. (2000). Predicting and understanding behavioral volitions: The interplay between goals and behaviors. European Journal of Social Psychology, 30, 705731. doi:10.1002/1099-0992(200009/10)30:5<705::AIDEJSP18>3.0.CO;2-\#

Povey, R., Conner, M., Sparks, P., James, R., \& Shepherd, R. (2000). The theory of planned behavior and healthy eating: Examining additive and moderating effects of social influence variables. Psychology \& Health, 14, 991-1006. doi:10.1080/08870440008407363

Sheeran, P., \& Orbell, S. (1999). Implementation intentions and repeated behavior: Augmenting the predictive validity of the theory of planned behavior. European Journal of Social Psychology, 29, 349-369. doi:10.1002/(SICI)10990992(199903/05)29:2/3<349::AID-EJSP931>3.0.CO;2-Y

Singelis, T. M., Triandis, H. C., Bhawuk, D., \& Gelfand, M. J. (1995). Horizontal and vertical dimensions of individualism and collectivism: A theoretical and measurement refinement. Cross-Cultural Research, 29, 240-275. doi:10.1177/106939719502900302

Sogn-Grundvåg, G., \& Østli, J. (2009). Consumer evaluation of unbranded and unlabelled food products: The case of bacalhau. European Journal of Marketing, 43, 213-228. doi:10.1108/03090560910923300

Tajfel, H. (1978). Social categorization, social identity, and social comparison. In H. Tajfel (Eds.), Differentiation between social groups: Studies in the social psychology of intergroup relations (pp. 61-67). London: Academic Press.

Toffler, A. (1980). The third wave. New York, NY: William Collins Sons \& Co. Ltd.

Triandis, HC., \& Singelis, T. M. (1998). Training to recognize individual differences in collectivism and individualism within culture. International Journal of Intercultural Relations, 22, 35-47. doi:10.1016/S0147-1767(97)00034-5

Verbeke, W., \& Vackier, I. (2004). Individual determinants of fish consumption: Application of the theory of planned behavior. Appetite, 44, 67-82. doi:10.1016/j.appet.2004.08.006

Xie, C., Bagozzi, R. P., \& Troye, S. (2008). Trying to prosume: Toward a theory of consumers as co-creators of value. Journal of the Academy of Marketing Science, 36, 109-122. doi:10.1007/s11747-007-0060-2

Zint, M. (2002). Comparing three attitude-behavior theories for predicting science teachers' intention. Journal of Research in Science Teaching, 39, 819-844. doi:10.1002/tea.10047

Correspondence regarding this article should be sent to: Richard P. Bagozzi, University of Michigan, 701 Tappan Street, Ann Arbor, MI 48109-1234 (bagozzi@umich.edu) 